\title{
Soluble biglycan: a potential mediator of cartilage degradation in osteoarthritis
}

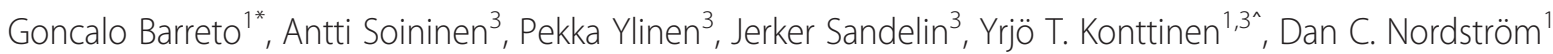 \\ and Kari K. Eklund ${ }^{2}$
}

\begin{abstract}
Background: Soluble biglycan (sBGN) and soluble decorin ( $(\mathrm{DCN})$, are two closely related essential components of extracellular matrix which both have been shown to possess proinflammatory properties. We studied whether sBGN or sDCN were present in synovial fluid (SF) of osteoarthritis (OA) or rheumatoid arthritis (RA) patients and studied sBGN or sDCN potential role in the degradation of OA cartilage.
\end{abstract}

Methods: SF obtained from meniscus tear, OA, and RA patients were analysed for sBGN and sDCN using enzyme-linked immunosorbent assays. OA chondrocytes and cartilage explants were stimulated for $48 \mathrm{~h}$ with $5 \mu \mathrm{g} / \mathrm{ml} \mathrm{sBGN} \mathrm{or} 1 \mu \mathrm{g} / \mathrm{ml}$ lipopolysaccharide. Messenger RNA (mRNA) levels of Toll-like receptors (TLRs), proteinases and cartilage matrix molecules were determined using quantitative real-time polymerase chain reaction. Protein levels of matrix metalloproteinases (MMPs) and cytokines were measured using Luminex xMap technology. Production of nitric oxide (NO), release of proteoglycans and soluble collagen were measured from conditioned culture media using biochemical assays. OA cartilage explant proteoglycans were stained for Safranin $\mathrm{O}$ and quantified using image analysis. TLR4 activation by SBGN and sDCN was studied in engineered HEK-293 cells with TLR4 signalling genes inserted together with a reporter gene.

Results: SBGN was found in meniscus tear SF (14 $2 \mathrm{ng} / \mathrm{ml})$, OA SF $(582 \pm 307 \mathrm{ng} / \mathrm{ml})$ and RA SF $(1191 \pm 482 \mathrm{ng} / \mathrm{ml})$. Low levels of sDCN could also be detected in SF of meniscus tear $(51 \pm 4) \mathrm{ng} / \mathrm{ml}, \mathrm{OA}(52 \pm 3 \mathrm{ng} / \mathrm{ml})$, and RA (49 $\pm 4 \mathrm{ng} / \mathrm{ml})$. Stimulation of chondrocytes with sBGN increased significantly the mRNA and protein expression of catabolic MMPs, including MMP1, MMP9 and MMP13, and of inflammatory cytokines interleukin (IL)-6 and IL-8, whereas the expression of anabolic markers aggrecan and collagen type II was decreased. sBGN induced release of proteoglycans, collagen and NO from chondrocytes and cartilage explants. The catabolic response in explants was dependent of OA cartilage degradation stage. The mechanism of action of sBGN was mainly mediated through the TLR4-nuclear factor-kB pathway.

Conclusions: High levels of sBGN was found in advanced OA and RA SF. sBGN activates chondrocytes mainly via TLR4, which results in net loss of cartilage. Thus, sBGN can be a mediator of OA cartilage degradation and also a potential biomarker for arthritis.

Keywords: Biglycan, Decorin, Osteoarthritis, Toll-like receptors, Chondrocytes, Cartilage, Innate immune response

\footnotetext{
* Correspondence: goncalo.barreto@helsinki.fi

Deceased

${ }^{1}$ Department of Internal Medicine and Rehabilitation, University of Helsinki

and Helsinki University (Central) Hospital, Biomedicum 1, PO Box 63,

FIN-00290 Helsinki, Finland

Full list of author information is available at the end of the article
} 


\section{Background}

Osteoarthritis (OA) is among the top five leading causes of disability worldwide [1]. To date, no effective longterm disease-modifying treatment for $\mathrm{OA}$ is available. At the root of the OA burden lies poor comprehension of the molecular pathophysiology of pre-symptomatic and clinically symptomatic OA.

Cartilage degeneration, a hallmark of OA, has its onset in wear, tear and mechanical injuries of the joint cartilage. Although trauma is perhaps the first causal event in $\mathrm{OA}$, the host inflammatory response plays an important role in the pathogenesis OA and inflammation is believed to be the major driver to symptomatic OA $[2,3]$.

Cells of the cartilage, known as chondrocytes, maintain cartilage tissue homeostasis. They control the structural assembly of extracellular matrix (ECM) and regulate destructive, remodelling and reparative processes [4]. In OA, cartilage-resident chondrocytes produce proteinases such as aggrecanases (ADAMTS-4, ADAMTS-5), matrix metalloproteinases (e.g., MMP-13 and MMP-9) and cathep$\sin \mathrm{K}$, and the anabolic synthesis of structural molecular components aggrecan and collagen type II (Col-II) are compromised [5]. Such a metabolic imbalance results in a failure of cartilage homeostasis and pathological cartilage destruction as well as eventual loss of cartilage.

ECM molecules have been thought to function as purely structural matrix-bound components, but evidence is emerging that they can also function as soluble ligands for pattern-recognising danger signalling receptors, mainly for Toll-like receptors (TLRs) [6-8]. Several of the ECM molecules found in OA joint and synovial fluid (SF) have been shown to trigger catabolic responses in TLR-equipped OA chondrocytes [9-11]. Biglycan (BGN) and decorin (DCN) are two small, closely related structural proteoglycans with leucine-rich repeats (small leucine-rich proteoglycan, SLRP) [12, 13]. The soluble forms of BGN (sBGN) or (sDCN) released from the cartilage matrix as a result of tissue injury could potentially function as an endogenous danger signal [14]. For example, sBGN, the size of which is approximately $95 \mathrm{kDa}$, has been shown trigger TLR4/TLR2 signalling in human aortic valve and in acute ischemic kidney, as well as to activate the NLRP3 inflammasome in macrophages [15-17]. sDCN has been shown to activate TLR4/ TLR2 signalling in macrophages [18].

We hypothesised that BGN is a prototypic example of a major essential cartilage ECM component. Upon release from stressed cartilage, it might activate TLRmediated catabolic signalling pathways $[19,20]$. In fact, several studies have shown that de novo BGN and DCN synthesis is increased in OA, that BGN and DCN fragments are produced and released by ADAMTSs and MMPs, and that OA SF also contains BGN and DCN autoantibodies [21-25]. However, to our knowledge, no studies have addressed the concentration of intact sBGN and $\mathrm{SDCN}$ in OA or rheumatoid arthritis (RA) SF or included examination of the eventual direct role of these proteoglycans in chondrocyte-mediated cartilage degradation in $\mathrm{OA}$.

Our aim was to study whether sBGN and sDCN can be found in SF from arthritic joints and whether sBGN and $\mathrm{sDCN}$ can contribute to cartilage degradation by activating the TLR-mediated catabolic signalling in primary OA chondrocytes and OA tissue explants.

\section{Methods}

\section{Tissue acquisition, cartilage explant culture and primary} chondrocyte culture

Patient recruitment and participation and sample collection were approved by the Helsinki and Uusimaa Hospital District ethics committee (Dnr_59/13/03/02/2013) and hospital board of directors (9310/407), and signed informed consent was obtained from the patients. Samples were collected from patients with $\mathrm{OA}$ who underwent total knee arthroplasty (TKA) $(\mathrm{N}=12)$. Half of the patients were women. The mean age of the patients was 63.5 years (range 53-73). Tibial plateau containing cartilage and subchondral bone was cut off to provide space for the tibial component of the TKA implant. Tibial plateau samples were collected into sterile sample containers containing cold phosphate-buffered saline solution (PBS) and processed within $2 \mathrm{~h}$.

Before tissue samples were harvested, the macroscopic appearance of the visible pathological changes in different sampling areas was recorded according to the arthroscopic grading scale of Société Française d'Arthroscopie [26]. Explant samples were collected from two different types of areas: grade I OA samples with no macroscopically visible lesions (which represents early OA cartilage degradation stages) or grade IV samples with full-thickness osteochondral lesions [27]. This grading of the samples makes possible a stratified analysis based on the degree of OA cartilage, from very mild to severe. Cylindrical full-thickness cartilage explants were obtained from graded tibial plateau areas using a 4-mm circular punch biopsy blade (Kai Medical, Oyana, Japan). Explants were cultured in Gibco Dulbecco's modified Eagle's medium (DMEM)/F-12 (Life Technologies, Carlsbad, CA, USA) supplemented with $10 \%$ foetal calf serum (FCS) for $24 \mathrm{~h}$ before stimulation.

When cartilage explants were not isolated, cartilage tissue (with bone cut away) was used for chondrocyte isolation. Chondrocytes form only $1-3 \%$ of the volume of the cartilage, so the whole cartilage was used for chondrocyte isolation to obtain enough cells. This means that the chondrocytes represent a heterogeneous population of OA chondrocytes. This does not allow a stratified analysis. Cartilage specimens were sequentially digested 
in $2.5 \mathrm{mg} / \mathrm{ml}$ pronase and $250 \mathrm{mg} / \mathrm{ml}$ collagenase $\mathrm{P}$ (Roche, Basel, Switzerland), with a PBS wash in between, for 60 minutes and overnight, respectively, under slow agitation at $37{ }^{\circ} \mathrm{C}$. The resulting cell suspension was filtered through a $70-\mu \mathrm{m}$ nylon cell strainer, centrifuged, washed twice with PBS and seeded at $1.5 \times 10^{5} / \mathrm{cm}^{2}$ in Gibco DMEM/F-12 supplemented with $10 \%$ FCS, $100 \mathrm{U} / \mathrm{ml}$ penicillin, $100 \mathrm{U} / \mathrm{ml}$ streptomycin and $0.25 \mu \mathrm{g} / \mathrm{ml}$ amphotericin B (Life Technologies). Cells were balanced for 3-4 days before stimulation. For cryopreservation, cells were suspended in FCS containing $10 \%$ (vol/vol) dimethyl sulphoxide and frozen in an isopropanol container for $24 \mathrm{~h}$ at $-80^{\circ} \mathrm{C}$ before storage in liquid nitrogen.

\section{Arthritis group definitions for synovial fluid collection Early osteoarthritis}

SF samples were collected from ten patients scheduled for arthroscopic surgery. The main indication for surgery was a suspicion of a meniscal tear. Patients with inflammatory arthritis, severe arthritis, corticosteroid injection within 6 weeks, blood dyscrasias or active malignancy were excluded. The prior use of nonsteroidal anti-inflammatory drugs was not considered an exclusion criterion. The diagnosis of early arthritis was made during arthroscopy and based on the presence of minimal visible chondral lesions.

\section{Advanced osteoarthritis}

SF samples were collected from ten patients scheduled for elective total knee replacement for management of primary idiopathic OA. The exclusion criteria were similar to those for patients with early OA.

\section{Rheumatoid arthritis}

SF samples were collected from ten patients with RA diagnosed fulfilling the American College of Rheumatology/ European League Against Rheumatism classification criteria for RA [28].

\section{Synovial fluid sample collection}

Patients with early OA, advanced OA and RA were, depending on the schedule of the surgeon, randomly selected for collection of SF samples via needle aspiration before opening of the joint. Blood-contaminated samples were excluded. SF maintained at $4{ }^{\circ} \mathrm{C}$ was aliquoted within $2 \mathrm{~h}$ into sterile Eppendorf tubes, centrifuged at $1200 \mathrm{~g}$ for 5 minutes at room temperature to separate solid debris and cells from the fluid phase, snap-frozen in liquid nitrogen and stored at $-80{ }^{\circ} \mathrm{C}$. When first thawed, SF was treated with a protease inhibitor cocktail (Roche Diagnostics, Meylan, France).

\section{Enzyme-linked immunosorbent assay}

SF was measured for intact-only sBGN and DCN molecules using a specific sandwich enzyme-linked immunosorbent assay (ELISA) (Uscn Life Science Inc., Hubei, China, and BioVendor Laboratorní medicína, Brno, Czech Republic, respectively) for detection of intact $\mathrm{sBGN}$ and sDCN molecules. BGN and DCN fragments are not detected by the immunoassays. Absorbance was measured at $450 \mathrm{~nm}$, as well as $450 \mathrm{~nm}$ and $630 \mathrm{~nm}$, for sBGN and sDCN immunoassays, respectively. All measurements were performed in duplicates.

\section{SEAP NF-KB activity assays}

TLR4 activity was measured using a cell-based assay according to the manufacturer's instructions (InvivoGen, San Diego, CA, USA). HEK-hTLR4 cells express TLR4 and $M D-2 / C D 14$ co-receptor genes of human origin and contain the secreted embryonic alkaline phosphatase $(S E A P)$ reporter gene for monitoring nuclear factor (NF) $-\kappa B$ activation. Upon interaction with the appropriate ligand, TLR4 transduces a signal which results in NF- $\mathrm{kB}$ activation and the expression of secreted alkaline phosphatase (AP), which can be detected by using detection medium (QUANTI-Blue, a medium used for the detection and quantification of secreted AP; InvivoGen) and measured with a spectrophotometer.

Briefly HEK-hTLR4 cells were cultured at a density of $2.5 \times 10^{4}$ cells in 96-well plates and maintained in complete DMEM with selective antibiotics, as described in the manufacturer's instructions (InvivoGen). Cells were then stimulated with $5 \mu \mathrm{g} / \mathrm{ml} \mathrm{BGN} \mathrm{(Sigma-Aldrich,} \mathrm{St.} \mathrm{Louis,} \mathrm{MO,}$ USA), $8 \mu \mathrm{g} / \mathrm{ml}$ sDCN (R\&D Systems, Minneapolis, MN, USA) or $1 \mu \mathrm{g} / \mathrm{ml}$ lipopolysaccharide (LPS) (positive control) for $24 \mathrm{~h}$, and activation of TLR4 and NF- $\mathrm{kB}$ signalling was analysed by measuring SEAP from conditioned culture medium samples according to the manufacturer's instructions (InvivoGen).

\section{Stimulation of chondrocyte and cartilage explant cultures} Primary chondrocytes cultures from passage 1 were stimulated for 24 or $48 \mathrm{~h}$ with $5 \mu \mathrm{g} / \mathrm{ml}$ bovine BGN (high sequence homology and structural conservation with human $\mathrm{BGN}$ ) obtained from articular cartilage and solubilised according to the manufacturer's instructions (Sigma-Aldrich) [29]. BGN endotoxin (LPS) contamination was not detected using the limulus amebocyte lysate test. As additional endotoxin controls, $5 \mu \mathrm{g} / \mathrm{ml}$ sBGN was incubated in fresh Gibco DMEM/F-12 containing $50 \mu \mathrm{g} / \mathrm{ml}$ proteinase $\mathrm{K}$ (Thermo Scientific, Waltham, MA, USA) or $25 \mu \mathrm{g} / \mathrm{ml}$ polymyxin B (InvivoGen) at $37{ }^{\circ} \mathrm{C}$ for $1 \mathrm{~h}$, after which the mixtures were added to the primary chondrocyte cultures. Protein homogeneity was confirmed by sodium dodecyl sulphate-polyacrylamide gel electrophoresis and Coomassie staining (performed by the manufacturer). LPS (Sigma-Aldrich) stimulation at $1 \mu \mathrm{g} / \mathrm{ml}$ was used as a positive TLR2/TLR4 control. In selected experiments, 1 MM CLI-095 (InvivoGen) was added 
to primary chondrocyte cultures 30 minutes before sBGN or LPS. CLI-095, also known as TAK-242, specifically suppresses TLR4 signalling mediated by the intracellular domain of the receptor. Further receptor-blocking studies were performed with the addition of $20 \mu \mathrm{g} / \mathrm{ml}$ TLR2 (Santa Cruz Biotechnology, Santa Cruz, CA, USA) or $20 \mu \mathrm{g} / \mathrm{ml}$ TLR4 (Santa Cruz Biotechnology) for $1 \mathrm{~h}$ before the addition of sBGN at $5 \mu \mathrm{g} / \mathrm{ml}$.

The optimal concentration for sBGN stimulation was determined in 24- and 48-h pilot experiments using $0.05,0.5$ and $5 \mu \mathrm{g} / \mathrm{ml}$ of sBGN, followed by analysis of MMP13, CTSK (cathepsin K, cat K), IL6 (interleukin-6, or IL-6) and COL2A1 (collagen type II $\alpha$ chain 1 , or ColIIA) gene messenger RNA (mRNA) copy numbers relative to the TATA box-binding protein (TBP) housekeeper, by using quantitative real-time polymerase chain reaction (qRT-PCR). Cartilage explant cultures were stimulated using the same conditions.

\section{Real-time polymerase chain reactions}

Total RNA was isolated from cartilage explants by using the RNAqueous ${ }^{\circ}$ kit (Thermo Scientific) and from primary chondrocytes by using the RNeasy ${ }^{\circ}$ Mini Kit (Qiagen, Valencia, CA, USA). Relative quantification of the gene levels was performed by comparing the cycle threshold $\left(C_{t}\right)$ values of the different genes, correcting for glyceraldehyde 3-phosphate dehydrogenase (GAPDH) and TBP content $\left(\Delta \mathrm{C}_{\mathrm{t}}\right)$ and for non-stimulated conditions $(\Delta \Delta \mathrm{Ct})$ and finally expressed as fold changes. Primer sequences are provided in Table 1.
Protein measurements using Luminex $\mathrm{xMAP}^{\circledR}$ technology Measurement of protein levels was done using $\mathrm{xMAP}^{\circ}$ technology (Luminex, Austin, TX, USA). To determine protein levels of MMPs (MMP-1, MMP-3, MMP-9 and MMP-13) as well as cytokines and chemokines (IL-6, IL8 ) in chondrocyte culture supernatant, $\mathrm{xMAP}^{\circ}$ technology on the Bio-Plex $200^{\circ}$ system (Bio-Rad Laboratories, Hercules, CA, USA) was used in combination with multiplex MMP/cytokine kits (ProcartaPlex; eBioscience; San Diego, CA, USA). Protein levels were measured in $25 \mu \mathrm{l}$ of culture medium (diluted 1:2).

\section{Measurement of nitric oxide}

Nitric oxide (NO) was measured from conditioned culture medium samples using a nitrate/nitrite colorimetric assay kit (Cayman Chemical, Ann Arbor, MI, USA). Nitrate was converted to nitrite by adding nitrate reductase and its cofactor, followed by the addition of Griess reagent to develop a deep purple colour. The absorbance was measured at $544 \mathrm{~nm}$ using a plate reader (Chameleon; Hidex, Turku, Finland).

\section{Measurement of soluble sulphated glycosaminoglycans and soluble collagen type II}

Soluble sulphated glycosaminoglycan (sGAG) standards, blanks and conditioned culture medium samples were mixed with the sGAG-binding Blyscan Dye Reagent ${ }^{\circ}$ for 30 minutes, followed by separation of the GAG-dye complex by centrifugation and dissociation of the dye from the pellet (Biocolor Ltd., Carrickfergus, UK). Soluble Col-II

Table 1 Primer pairs used for real-time polymerase chain reactions

\begin{tabular}{|c|c|c|}
\hline Gene & Protein name & Primer sequence \\
\hline \multirow[t]{2}{*}{ ADAMTS4 } & \multirow[t]{2}{*}{ A disintegrin and metalloproteinase with thrombospondin motifs 4} & Forward: 5'-AAT CCT GTC AGC TTG GTG GT-3' \\
\hline & & Reverse: 3'-CTT GGA GTT GTC ATG GAG CA-5' \\
\hline \multirow[t]{2}{*}{ ADAMTS5 } & \multirow[t]{2}{*}{ A disintegrin and metalloproteinase with thrombospondin motifs 5} & Forward: 5'-CTT CAC TGT GGC TCA CGA AA-3' \\
\hline & & Reverse: 3'-AAT GTC AGG TTG CAC TGC TG-5' \\
\hline \multirow[t]{2}{*}{ MMP13 } & \multirow[t]{2}{*}{ Matrix metalloproteinase 13 (MMP-13) } & Forward: 5'-CTA TGG TCC AGG AGA TGA AG-3' \\
\hline & & Reverse: 3'-AGA GTC TTG CCT GTA TCC TC-5' \\
\hline \multirow[t]{2}{*}{ CTSK } & \multirow[t]{2}{*}{ Cathepsin K } & Forward: 5'-ACC CAA CAG GCA AGG CAG CTAA-3' \\
\hline & & Reverse: 3'-GCA ATG CCA CAGG CGT TGT TCT-3' \\
\hline \multirow[t]{2}{*}{ MMP9 } & \multirow[t]{2}{*}{ Matrix metalloproteinase 9 (MMP-9) } & Forward: 5'-TTC TCC AGA AGC AAC TGT CC-3' \\
\hline & & Reverse: 3'-CGG CAA GTC TTC CGA GTA GT-5' \\
\hline \multirow[t]{2}{*}{ ACAN } & \multirow[t]{2}{*}{ Aggrecan } & Forward: 5'-TGG TGA TGA TCT GGC ACGA-3' \\
\hline & & Reverse: 3'-TCT GCG TIT GTA GGT GGTG-5' \\
\hline \multirow[t]{2}{*}{ COL2A1 } & \multirow[t]{2}{*}{ Collagen, type II, alpha 1} & Forward: 5'-GAG TCA AGG GTG ATC GTG GT-3' \\
\hline & & Forward: 5'-AAG CAC CTT GGT CTC CAG AA-5' \\
\hline \multirow[t]{2}{*}{ TBP } & \multirow[t]{2}{*}{ TATA box-binding protein } & Forward: 5'-GAA GAA CAA TCC AGA CTA GCA GCA-3' \\
\hline & & Reverse: 3'-CCT TAT AGG GAA CTT CAC ATC ACAG-5' \\
\hline \multirow[t]{2}{*}{ GAPDH } & \multirow[t]{2}{*}{ Glyceraldehyde 3-phosphate dehydrogenase } & Forward: 5'-AAG GTC ATC CCT GAG CTG AA-3' \\
\hline & & Reverse: 3'-TGC TGT AGC CAA ATT CGT TG-5' \\
\hline
\end{tabular}


was similarly mixed with the collagen-binding Sircol Dye Reagent ${ }^{\circ}$ (Biocolor Ltd.) for 30 minutes, followed by centrifugation, pelleting, washes with salt wash reagent, centrifugation and dissociation of the collagen-dye complex using an alkali reagent vortexing wash. Absorbance was measured at 595 and $540 \mathrm{~nm}$, respectively, using a plate reader (Chameleon).

\section{Safranin $O$ staining}

Control and sBGN-stimulated cartilage explants were fixed in neutral buffered $10 \%$ formalin for 2 weeks before dehydration in ethanol series, clearing in xylene and embedding in paraffin. Deparaffinised tissue sections were stained with Fast Green dye, rinsed in $1 \%$ acetic acid and stained in $0.1 \%$ Safranin $O$ before evaluation using a Nikon LV-DIA Base microscope (Nikon Instruments, Tokyo, Japan) with a motorized XY staging system (OptiScan III; Prior Scientific, Rockland, MA, USA) connected to a DS-Fi1 digital camera (Nikon Instruments) using NIS-Elements Basic Research analysis (Nikon Instruments). Image analysis was done using ImageJ software (National Institutes of Health, Bethesda, MD, USA).

\section{Statistical analysis}

Differences between groups were tested using the Mann-Whitney $U$ test, the Wilcoxon signed-rank test or Student's $t$ test, as appropriate. Effects of covariates were analysed by multiple linear regression. All statistical analysis were performed using IBM SPSS version 21 software (IBM, Armonk, NY, USA). $p$ Values $<0.05$ were considered significant.

\section{Results}

sBGN and SDCN can be found in synovial fluid obtained from patients with $O A$ or RA

BGN and DCN are key components of the cartilage matrix. We hypothesised that some intact BGN or DCN could be released from matrix into SF in OA or RA and that in soluble form they could act as a proinflammatory stimulus. Indeed, sandwich ELISA disclosed clearly higher
sBGN levels in advanced OA $(582 \pm 307 \mathrm{ng} / \mathrm{ml})$ than the levels found in patients with meniscus tear who had very early OA $(14 \pm 2 \mathrm{ng} / \mathrm{ml})$ (Table 2$)$. The highest levels of sBGN were observed in SF obtained from patients with RA $(1191 \pm 482 \mathrm{ng} / \mathrm{ml})$ (Table 2). In contrast to sBCN, the SF levels of sDCN were low, and no differences were observed between early OA $(51 \pm 4 \mathrm{ng} / \mathrm{ml}), \mathrm{OA}(52 \pm 3 \mathrm{ng} / \mathrm{ml})$ and RA: $(49 \pm 4 \mathrm{ng} / \mathrm{ml})$. Covariates (age, sex and body mass index) tested with multiple linear regression models were not associated with SF sBGN or SF sDCN levels.

\section{sBGN upregulates catabolic factors in OA chondrocytes}

As high concentrations of intact sBGN were found in the SF of OA and RA patients, we studied whether sBGN could have effects on the cartilage metabolism. First, we studied the effect of sBGN on the expression of catabolic cartilage factors. In primary monolayer chondrocytes, sBGN significantly increased gene expression of ADAMTS4, ADAMTS5, MMP13, CTSK and MMP9 almost as efficiently as LPS (Fig. 1a). Studies of cartilage explant tissues obtained from patients with varying degrees of OA severity revealed that the response to sBGN was dependent on the grade of cartilage degradation. sBGN induced pronounced expression of catabolic factors in grade I OA cartilage, whereas a more modest increase of catabolic factors was observed in highly degenerated grade IV OA cartilage (Fig. 1b).

To further assess whether the studied gene mRNA transcripts were also translated into proteins, we used Luminex $\mathrm{xMAP}^{\circ}$ technology. In cell culture supernatants, the levels of MMP-1 and MMP- 3 were substantially upregulated (1.6- and 3.6-fold, respectively) by sBGN compared with unstimulated controls (Fig. 2). Compared with positive control LPS, the effects of sBGN on protein levels for MMP-1, MMP-3, MMP-9 and MMP- 13 were $3 \%, 50 \%, 82 \%$ and $21 \%$, respectively, of that of LPS (LPS data not shown).

Levels of proinflammatory cytokines IL-6 and IL-8 were also upregulated by sBGN compared with unstimulated control (1.4- and 2.2-fold, respectively) (Fig. 2). Compared with LPS, the effects of sBGN on cytokine

Table 2 Intact sBGN and sDCN concentration levels in synovial fluid obtained from knee joints of early osteoarthritis, advanced osteoarthritis and rheumatoid arthritis patients

\begin{tabular}{llll}
\hline Characteristics & Early OA $(n=10)$ & Advanced OA $(n=10)$ & RA $(n=10)$ \\
\hline Patient age, yr & $63.1 \pm 16$ & $64.4 \pm 10$ & $55.3 \pm 15.4$ \\
Males & $40(4)$ & $40(4)$ & $30(3)$ \\
Body mass index, $\mathrm{kg} / \mathrm{m}^{2}$ & $27.2 \pm 4.4$ & $27.8 \pm 3.5$ & $24.3 \pm 4.7$ \\
$\mathrm{sBGN}, \mathrm{ng} / \mathrm{ml}$ & $14 \pm 2$ & $582 \pm 307^{\mathrm{a}}$ & $1191 \pm 482^{\mathrm{a}}$ \\
$\mathrm{sDCN}, \mathrm{ng} / \mathrm{ml}$ & $51 \pm 4$ & $52 \pm 3$ & $49 \pm 4$ \\
\hline
\end{tabular}

$O A$ osteoarthritis, $R A$ rheumatoid arthritis, $s B G N$ soluble biglycan, $s D C N$ soluble decorin Data are presented as mean \pm SD or count (\%)

${ }^{\mathrm{a}} p<0.01$ by Student's $t$ test 
A catabolic factors mRNA - primary chondrocytes

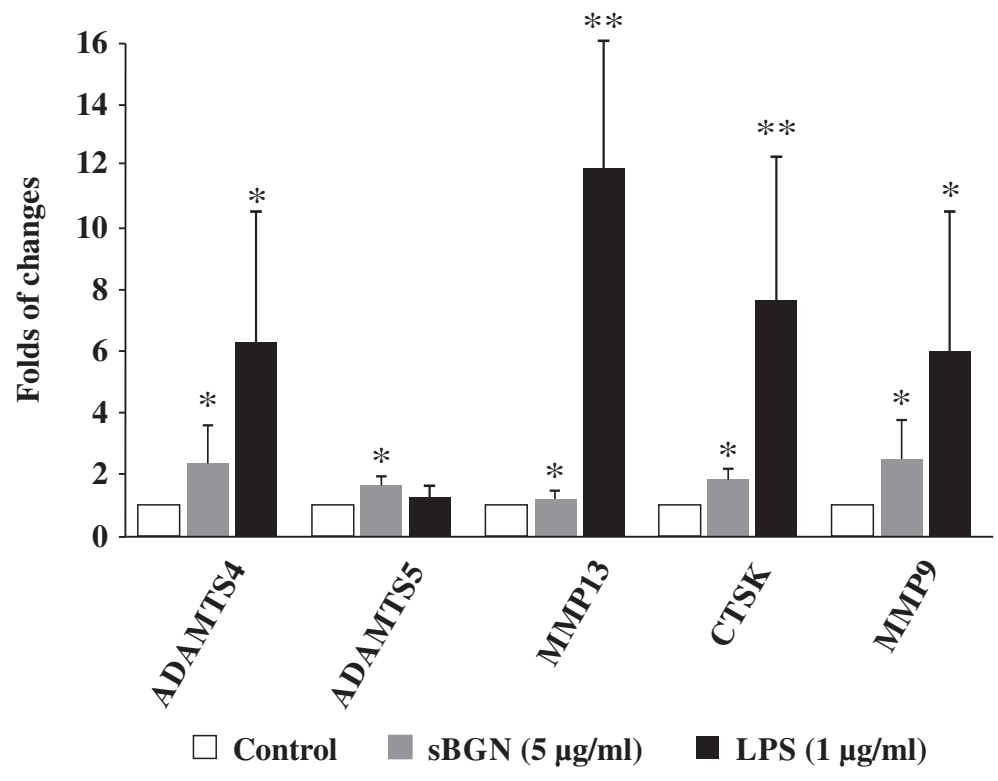

B catabolic factors mRNA - explants

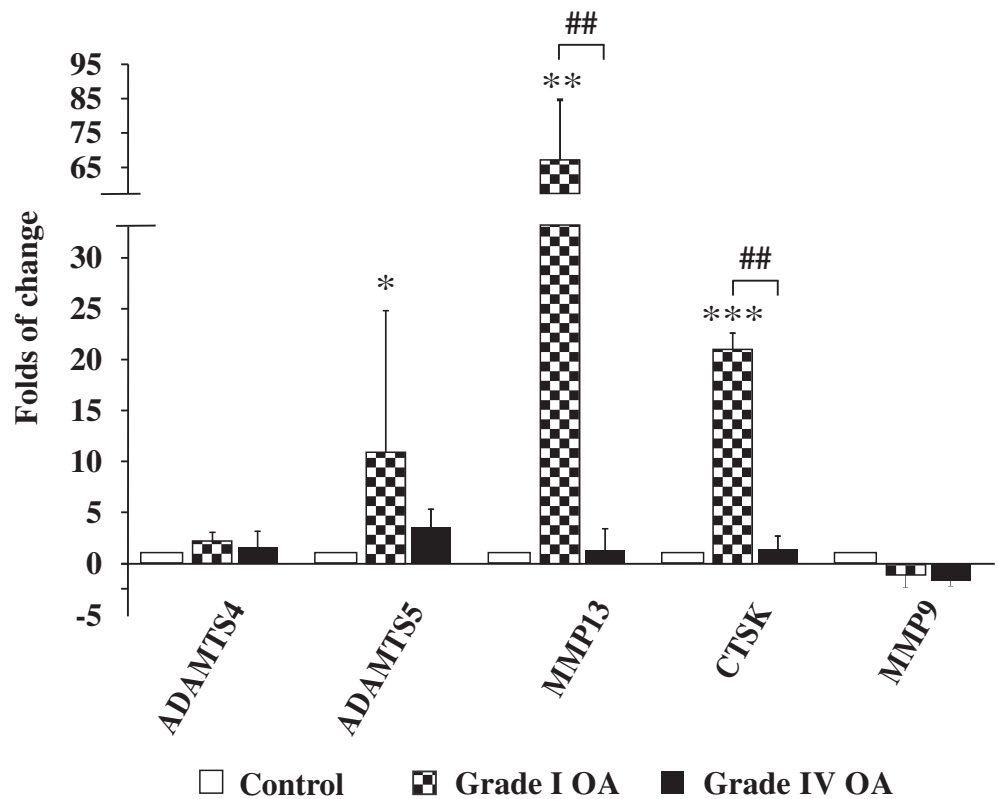

Fig. 1 Effect of soluble biglycan (sBGN) on the expression of proteinases associated with matrix remodelling and cartilage degeneration in osteoarthritis (OA). a In primary chondrocytes, sBGN increased all proteinases examined. Lipopolysaccharide (LPS) was used as a positive control. b In cartilage explants, SBGN increased ADAMTS5, MMP13, CTSK and MMP9 messenger RNA (mRNA) only in mild grade I OA and not in severe grade IV OA. The results are expressed as fold changes (mean \pm SD) relative to TATA box-binding protein (TBP) housekeeper and compared with non-stimulated controls. Samples were run as technical duplicates, and each experiment was done using samples obtained from at least six different donors (biological replicates). ${ }^{*} p<0.05,{ }^{* *} p<0.01,{ }^{* * *} p<0.001$ vs. non-stimulated controls; ${ }^{* \#} p<0.01$ for pairwise comparisons between mild grade I OA and severe grade IV OA. ADAMTS a disintegrin and metalloproteinase with thrombospondin type 1 motif (aggrecanase), MMP matrix metalloproteinase, CTSK cathepsin K 


\section{MMPs and cytokines protein levels in chondrocyte cell culture supernatant}
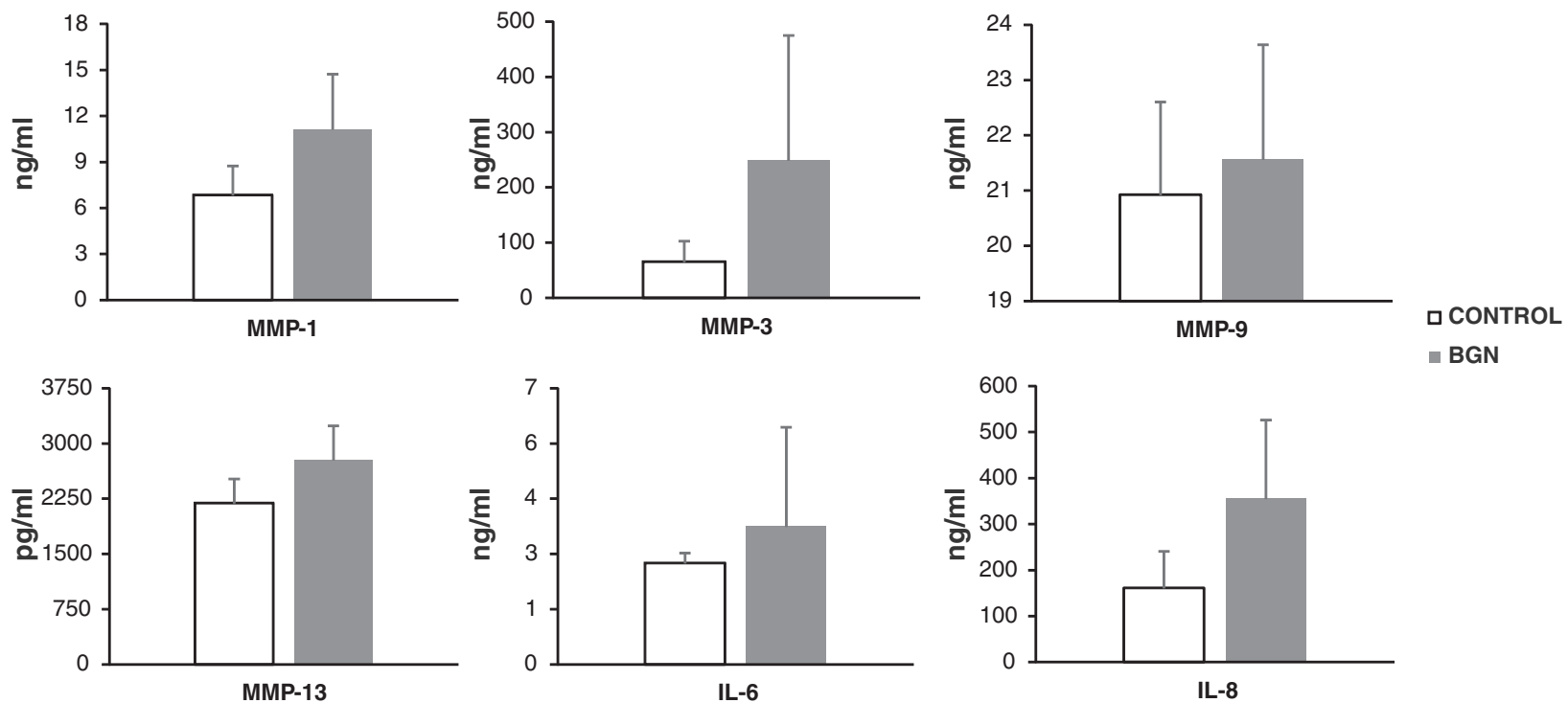

Fig. 2 Effect of soluble biglycan (sBGN) on the expression of matrix metalloproteinase (MMP)-1, MMP-3, MMP-9 and MMP-13 as well as interleukin (IL)-6 and IL-8 proteins. sBGN leads to the upregulation chondrocyte-mediated production of major catabolic markers known in osteoarthritis. SBGN-induced concentration levels compared with controls did not reach the statistical significance level $<0.05$. The results are expressed mean \pm SD. Samples were run as technical duplicates, and each experiment was done using samples from three different donors (biological replicates)

levels for IL-6 and IL-8 were $18 \%$ and $25 \%$, respectively (LPS data not shown).

\section{sBGN impairs the synthesis of major cartilage matrix components in $\mathrm{OA}$ chondrocytes}

To determine the effect of sBGN on overall cartilage turnover, we studied the effect of sBGN on the anabolic functions of OA chondrocytes. In primary OA chondrocytes, sBGN significantly inhibited aggrecan and Col-II mRNA expression (Fig. 3a). The studies on cartilage explants revealed that sBGN significantly decreased Col-II mRNA expression in highly degenerated grade IV OA but had no significant effect on the expression in grade I OA cartilage (Fig. 3b).

\section{sBGN increases proteoglycan and collagen release from cartilage explants}

Release of proteoglycans and soluble collagen from cartilage explants into cell culture media was determined to confirm that $\mathrm{sBGN}$ can also induce the degradation of OA cartilage by catabolic chondrocytes in situ. sBGN induced prominent release of sGAG in grade I OA explants, whereas sBGNinduced collagen release was observed only in grade IV OA cartilage (Fig. 4). Proteoglycan release was further confirmed by Safranin O staining in sBGN-treated cartilage explants. sBGN decreased the total quantity of proteoglycan in explants by approximately $50 \%$ (Fig. 5).
sBGN upregulates TLR4 expression in chondrocytes and in cartilage tissue explants

To study the mechanism of the effects of sBGN on cartilage metabolism, the effects of sBGN on TLR expression and function were first studied. Expression of TLR2 and TLR4 mRNA was observed in primary monolayer chondrocytes and cartilage explants of all patients with OA. In accordance with earlier studies, basal TLR2/TLR4 levels were slightly (1.3-fold) higher in grade IV OA than in grade I OA [30]. In primary monolayer chondrocyte cultures, sBGN significantly increased TLR4 expression but did not have any significant effect on TLR2 mRNA expression (Fig. 6a).

Studies of cartilage explants revealed that the TLR response to $\mathrm{sBGN}$ is dependent on the grade of cartilage degradation. In grade I OA, the sBGN-induced increase of TLR4 mRNA expression was $352 \%$ than that observed in grade IV OA cartilage (Fig. 6b). In contrast, there was no difference in sBGN-induced TLR2 mRNA expression between grade I and grade IV OA (Fig. 6b). Blocking TLR4 by using a low molecular weight inhibitor of TLR4 signalling (CLI095) abrogated the sBGNinduced increase of TLR4 mRNA expression (Fig. 7a).

\section{Catabolic effect of sBGN in articular chondrocytes is mediated via TLR4}

Inducible nitric oxide synthase (iNOS) is activated via TLR2/TLR4/NF-kB pathway activation. To further evaluate 


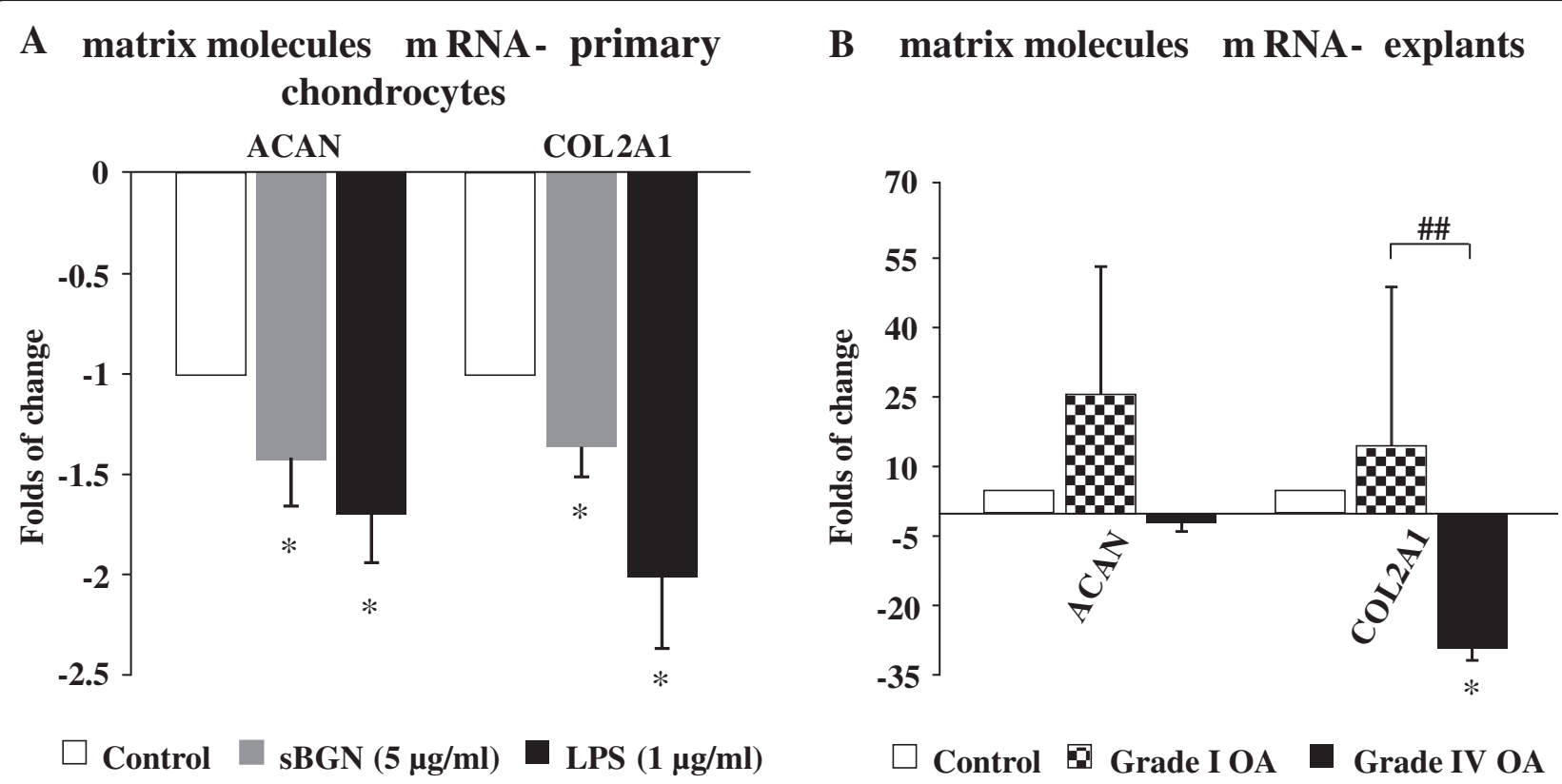

Fig. 3 Effects of soluble biglycan (sBGN) on the expression of aggrecan and collagen type II (Col-II). a In primary chondrocytes, sBGN decreased aggrecan and Col-II. Lipopolysaccharide (LPS) was used as a positive control. b In cartilage explants, sBGN did not affect aggrecan (ACAN) or Col$\|(C O L 2 A 1)$ in grade I osteoarthritis (OA) but decreased Col-I(COL2A1) in grade IV OA, with a different response in mild and severe OA. The results are expressed as fold changes (mean \pm SD) relative to the TATA box-binding protein (TBP) housekeeper and compared with non-stimulated controls. Samples were run as technical duplicates, and each experiment was done using samples obtained from at least six different donors (biological replicates). ${ }^{*} p<0.05$ vs. non-stimulated controls; ${ }^{\# \#} p<0.01$ for pairwise comparisons between mild grade I OA and severe grade IV OA. mRNA messenger RNA

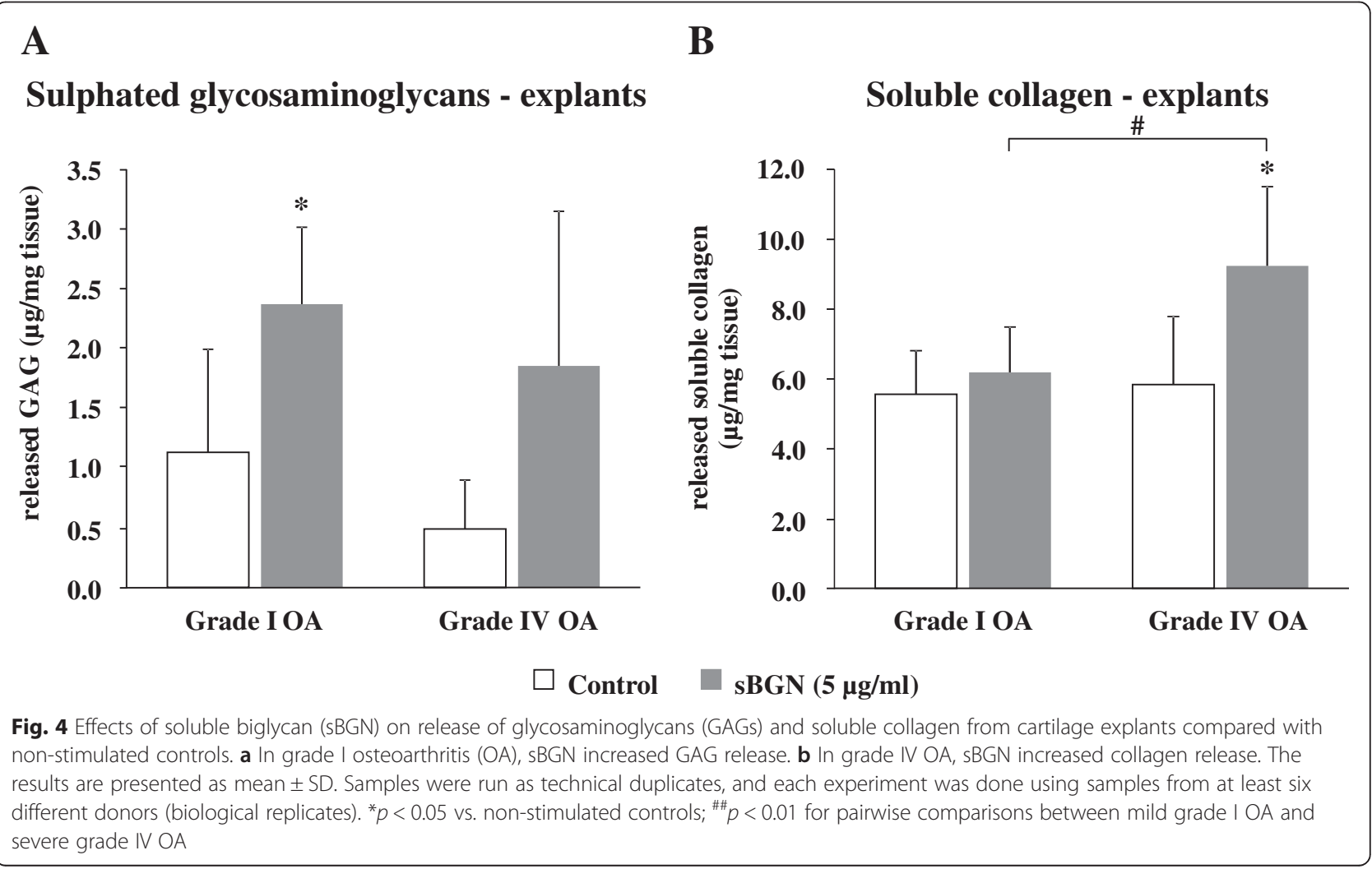




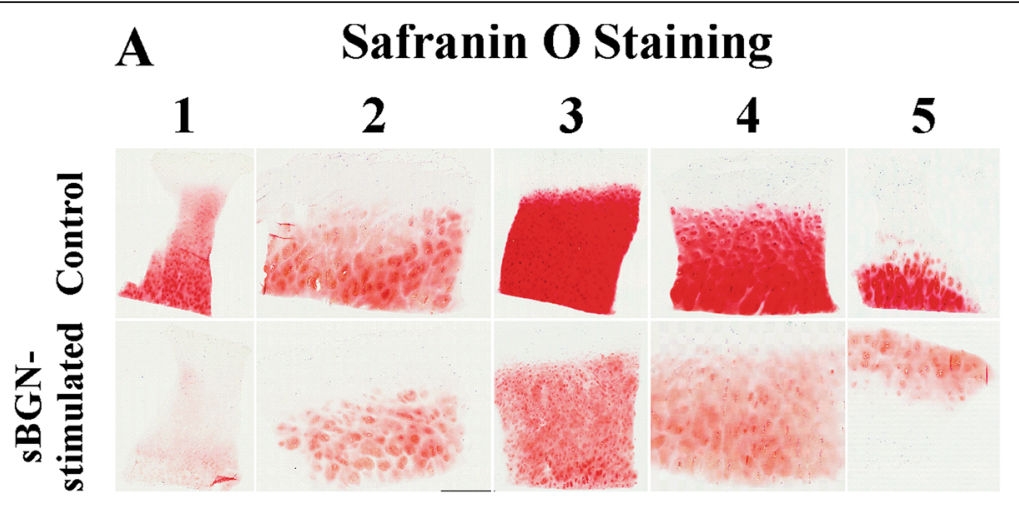

\section{B Safranin O staining quantification}

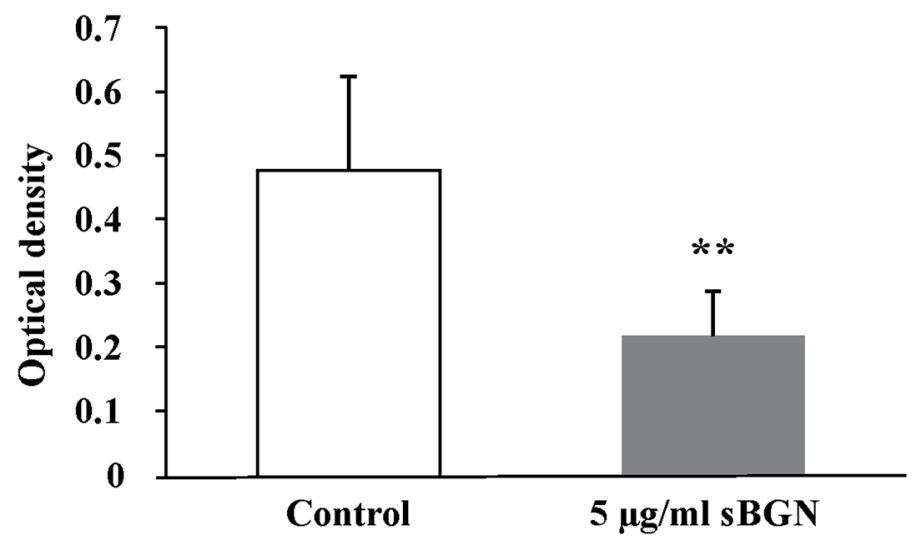

Fig. 5 Effects of soluble biglycan (sBGN) on proteoglycan content in osteoarthritis (OA) cartilage explants from five different patients. a Safranin 0 staining of the formalin-fixed, paraffin-embedded tissue sections produced from the OA cartilage explants (4 mm) after 48-h incubation. Note extensive proteoglycan depletion in all samples. Safranin O photomicrographs are panoramic images constructed from several photomicrographs to provide an overall view of cartilage explant extent in one image. Original magnification, $\times 20$. b Proteoglycan depletion was quantified by optical density of Safranin O staining using ImageJ image analysis software. The results are presented as mean \pm SD. Proteoglycan content was reduced by $50 \pm 20 \%$ compared with non-stimulated samples. ${ }^{* *} p<0.01$

the role of TLRs in sBGN-induced signalling, we studied the effect of sBGN on NO release from primary OA chondrocytes. In primary OA chondrocyte cultures, sBGN significantly increased NO production (Fig. 8a). In cartilage explant tissues, sBGN increased NO production slightly more in grade I OA than in grade IV OA (Fig. 8b). Blocking of TLR4 signalling with TLR4-neutralising antibody or synthetic inhibitor CLI-095 significantly reduced NO production to levels observed in non-stimulated controls (Fig. 8c), suggesting that the effect of sBGN is mediated via TLR4. To exclude the effect of contaminating LPS, polymyxin B was added, which had no effect on the sBGN-induced production of NO. In contrast, proteinase $\mathrm{K}$ reduced NO production to levels of non-stimulated controls, further supporting the finding that the observed effect of sBGN is not caused by LPS contamination. Moreover, blocking of TLR4 signalling abrogated the increase in
mRNA expression of the catabolic factors induced by both sBGN and LPS while also restoring to normal the levels of the mRNA expression of the key anabolic factors $A C A N$ and COL2A1 (Fig. 7b and c). In contrast to TLR4, TLR2neutralising antibody had no significant effect on NO production. Together, these above suggest that the effect of sBGN on cartilage metabolism is mediated via activation of TLR4 and that TLR2 has either no or only a minor role.

To confirm that $\mathrm{sBGN}$ signalling is mediated through TLR4/NF- $K B$ pathway, we studied the activation of NF$\kappa B$ after stimulation with sBGN. This was done using engineered HEK-hTLR4 cells, which can be stimulated only through activation of the TLR4 receptors. These cells have been stably transfected with SEAP plasmid containing NF- $\mathrm{KB}$ response elements, and the activation of NF- $\mathrm{kB}$ can be monitored by measuring activity of 


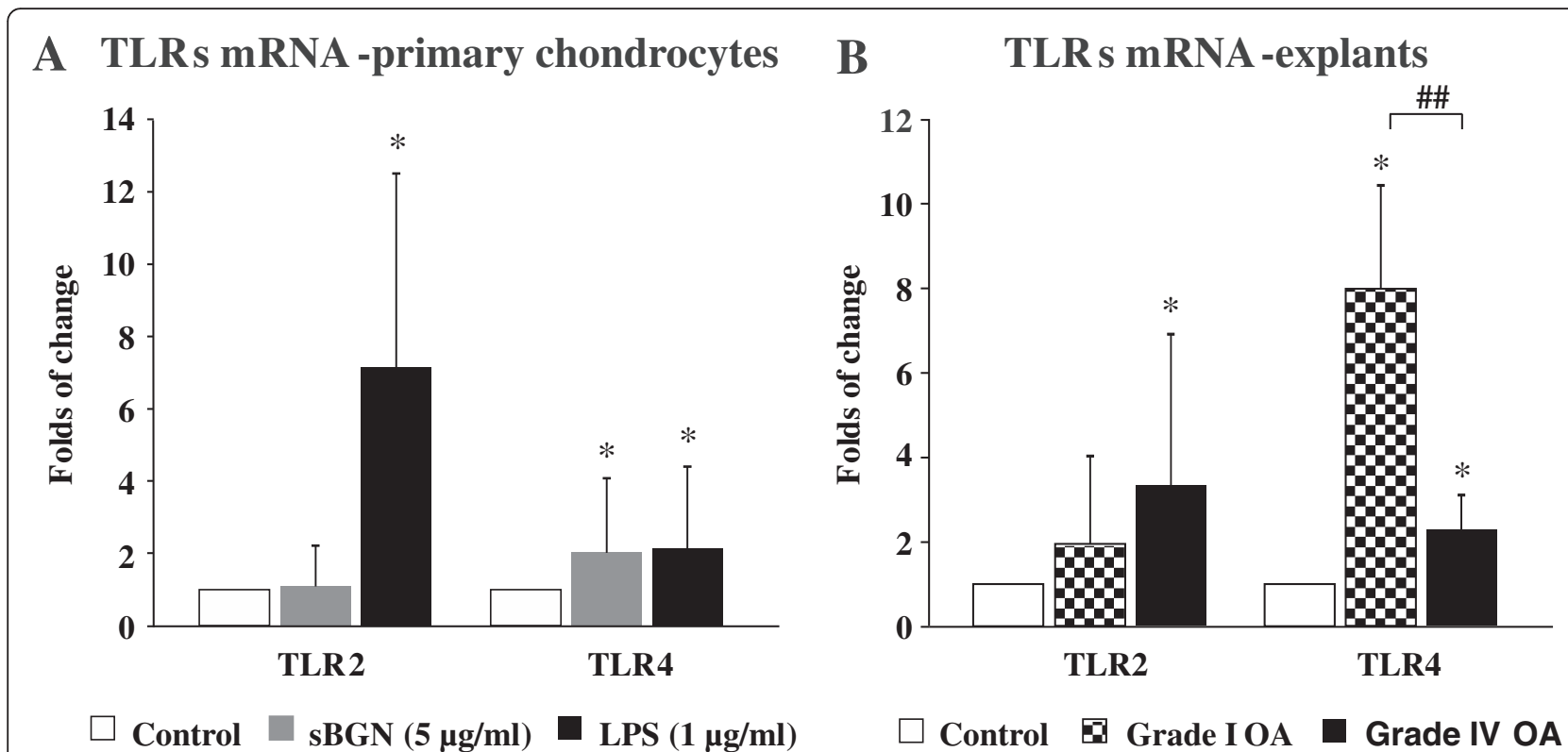

Fig. 6 Effect of soluble biglycan (sBGN) on expression of Toll-like receptor 2 (TLR2) and Toll-like receptor 4 (TLR4) on primary chondrocytes. a In primary chondrocytes, sBGN increased TLR4 $(p<0.05)$ and did it as effectively as lipopolysaccharide (LPS), which was used as a positive control. b In cartilage explants, sBGN increased TLR4, both in mild grade I $(p<0.05)$ and in severe grade IV $(p<0.05)$ osteoarthritis $(O A)$, but differently in mild and severe OA. The results are expressed as fold changes (mean \pm SD) relative to TATA box-binding protein (TBP) housekeeper and compared with non-stimulated controls, which corresponds to a fold change of 1. Samples were run as technical duplicates, and each experiment was done using samples obtained from at least six different donors (biological replicates). ${ }^{*} p<0.05$ vs. non-stimulated controls; ${ }^{\# \#} p<0.01$ for pairwise comparisons between mild grade I OA and severe grade IV OA

SEAP. Stimulation of HEK-hTLR4 cells with sBGN caused a substantial increase of SEAP, while inhibition of TLR4 signalling with TLR4 inhibitor CLI-095 prevented the increase of SEAP levels. SDCN and LPS caused a similar increase of SEAP levels. Proteinase $\mathrm{K}$ abrogated SEAP upregulation by sBGN and SDCN, while polymyxin $\mathrm{B}$ did not alter the response, thus ensuring that the studied effect was not caused by endotoxin contamination (Fig. 8d).

\section{Discussion}

BGN and DCN are two major non-collagenous SLRP products of chondrocytes which are deposited into articular cartilage matrix [13]. In this study, we show, for the first time to our knowledge, that intact $\mathrm{sBGN}$ is present in SF obtained from patients with meniscus tear lesions and early OA, advanced OA or RA, with the highest concentrations found in advanced OA and RA. DCN could also be found in SF, but, in contrast to BGN, only low levels were detected and no differences between early OA, advanced OA and RA were observed. Thus, matrix-embedded chondrocytes as well as other cells in joints are exposed to fluid phase sBGN and sDCN. Intact sBGN and sDCN, but not their fragments, have been shown to engage proinflammatory responses $[31,32]$. Therefore, while BGN fragments are known to be released into the $\mathrm{SF}$ of patients with $\mathrm{OA}$, their presence is merely a reflection of disease activity. Hence, they have been proposed to be solely a diagnostic biomarker. The observed increased levels of intact sBGN are also in line with the observed upregulation of mRNA and protein levels of sBGN in human OA cartilage tissue and also in cartilage obtained using a sheep meniscectomy animal model of OA. Although DCN mRNA and protein levels have been shown to increase in OA, this was not reflected in the level of DCN in SF [22, 25, 33]. Interestingly, SF of OA and RA patients has been shown to contain immunoglobulin $G$ autoantibodies against BGN, DCN and several other cartilage matrix molecules, suggesting their release from matrix and subsequent local loss of immunological tolerance [34]. Immune complex formation leading to complement and $\mathrm{Fc} \gamma$ receptor activation plays a role in RA, as well as in OA according to recent studies [35-37]. The present finding of intact $\mathrm{sBGN}$ in $\mathrm{SF}$, together with the recognition of sBGN as an endogenous damage-associated molecular pattern-type ligand for TLR2/TLR4 and for $\mathrm{P}_{2} \mathrm{X}_{7 / 4}$, provides a third mechanism of relevance for autoinflammation, co-stimulation and autoimmunity.

Chondrocytes of OA cartilage are equipped with a full palette of TLRs, including TLR2 and TLR4, and the proportion of $\mathrm{TLR}^{+}$chondrocytes increases with progression of OA [38]. The results of the present study show that $\mathrm{sBGN}$ regulates TLR4 expression to an extent 
A TLRs mRNA - primary chondrocytes
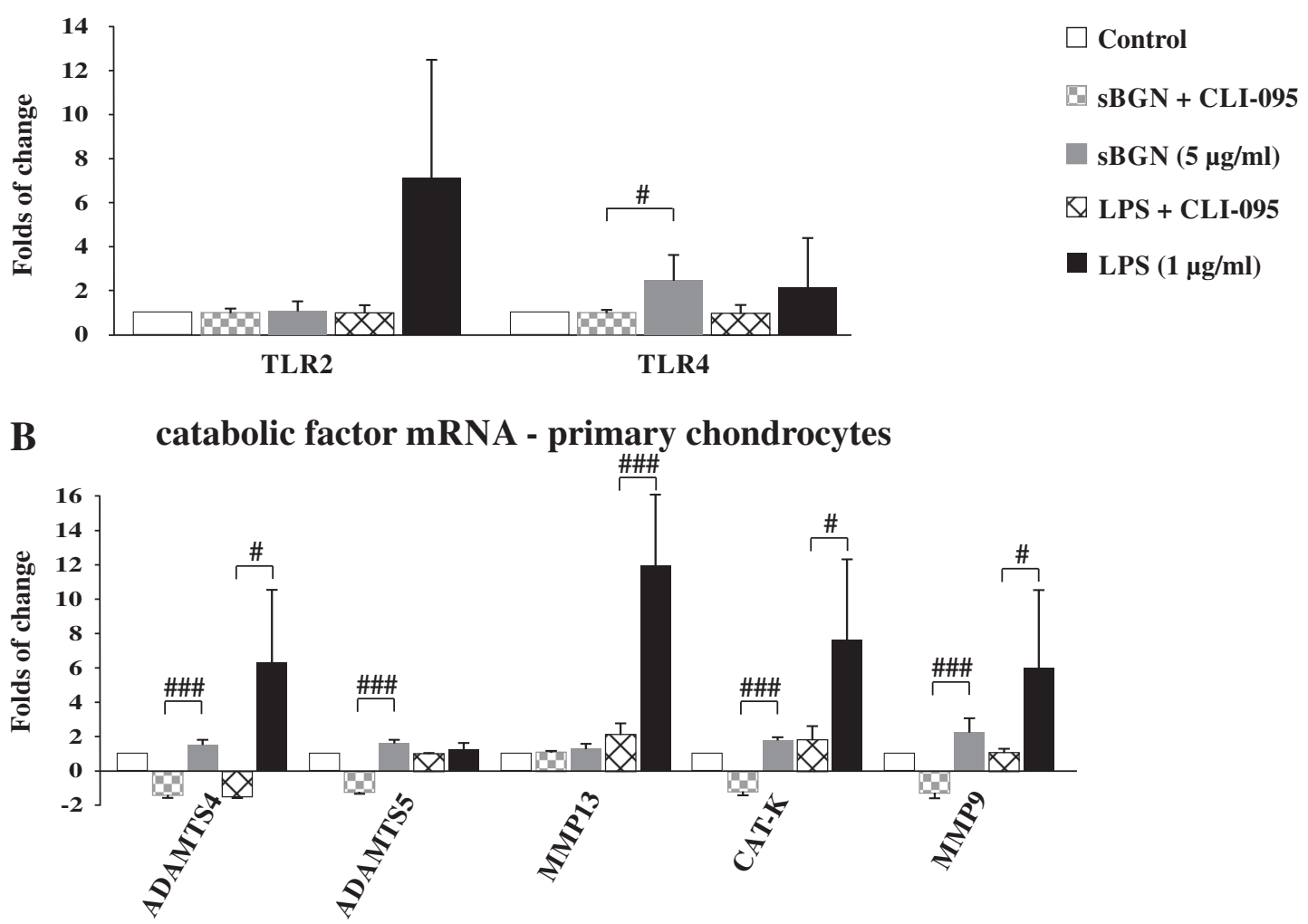

C catabolic factor mRNA - primary chondrocytes

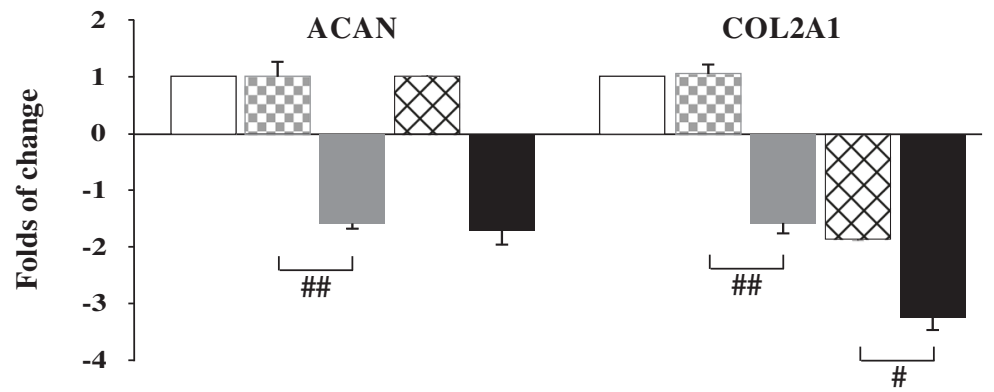

D Nitric Oxide release - primary chondrocytes

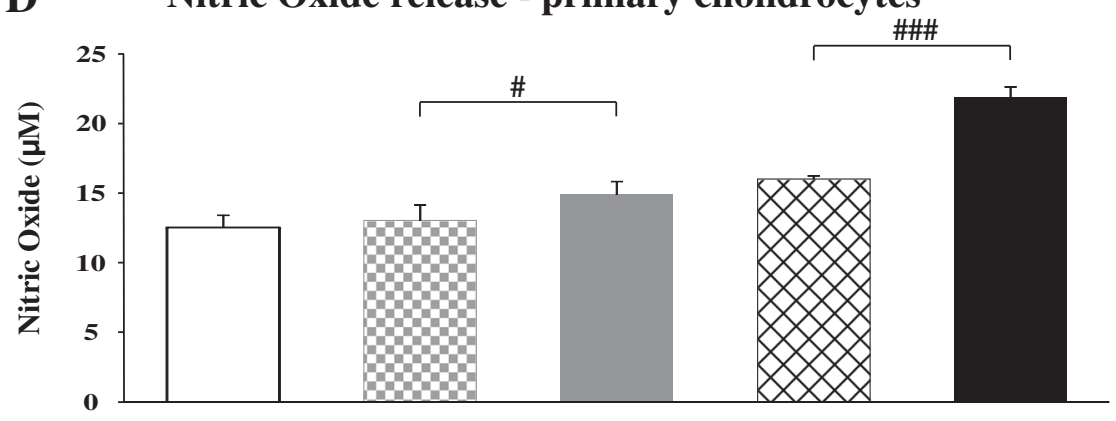

Fig. 7 (See legend on next page.) 
(See figure on previous page.)

Fig. 7 CLI-095 abrogates soluble biglycan (sBGN)- and lipopolysaccharide (LPS)-induced Toll-like receptor 4 (TLR4) signalling with CLI-095 in primary chondrocytes. a Pre-incubation with CLI-095 before sBGN and LPS stimulation abrogated the increase in TLR4 messenger RNA (mRNA) expression. b A minor increase in catabolic factor mRNA expression occurred when primary chondrocytes were treated with CLI-095 before sBGN and LPS stimulation. c Blocking TLR4 signalling with CLI-095 inhibited the sBGN-induced upregulation of aggrecan (ACAN or ADAMTS) and collagen type II (COL2A1) before stimulation. $\mathbf{d}$ Production of nitric oxide is reduced by CLI-095 incubation before sBGN or LPS stimulation. All in vitro experiments were technically repeated at least four times with primary chondrocytes (obtained from one biological sample). Differences between mean values were compared using Student's $t$ test, assuming normal distribution where four replicates were used. ${ }^{\#} p<0.05,{ }^{\# \#} p<0.01,{ }^{\# \#} p<0.001$ for pairwise comparisons between CLI-095 preincubated and non-CLI-095 pre-incubated sBGN or LPS stimulation. CTSK cathepsin K, MMP matrix metalloproteinase

similar to that of LPS, which is a known TLR2/TLR4 ligand. The effects of sBGN were not caused by LPS contamination, as the result of the endotoxin test was negative and polymyxin $\mathrm{B}$ did not have any effect on the results in HEK-hTLR4 cells or in primary chondrocytes.
Thus, sBGN can be conceived of as an endogenous damage-associated molecular pattern, a cartilage-derived LPS mimic. The mechanism of action of sBGN and DCN is mediated through activation of TLR4 and the NF-kB pathway. This is supported by several lines of

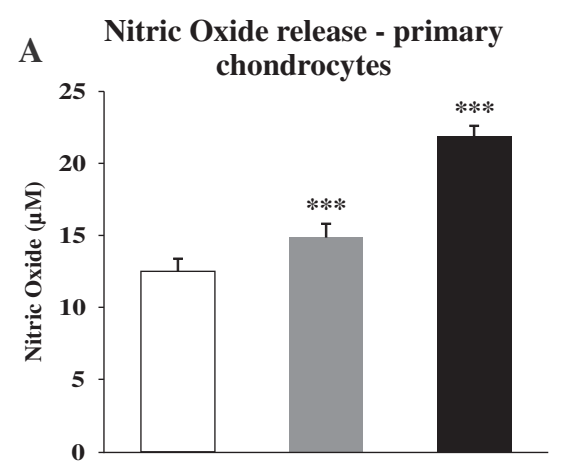

B

B Nitric Oxide release - explants

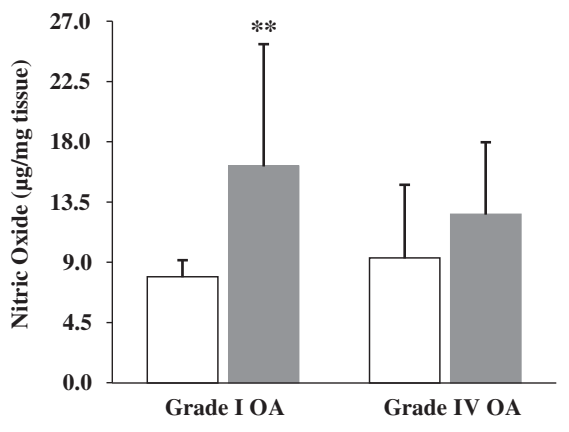

$\square$ Control $\square \operatorname{sBGN}(5 \mu \mathrm{g} / \mathrm{ml}) \quad \square \operatorname{LPS}(1 \mu \mathrm{g} / \mathrm{ml})$
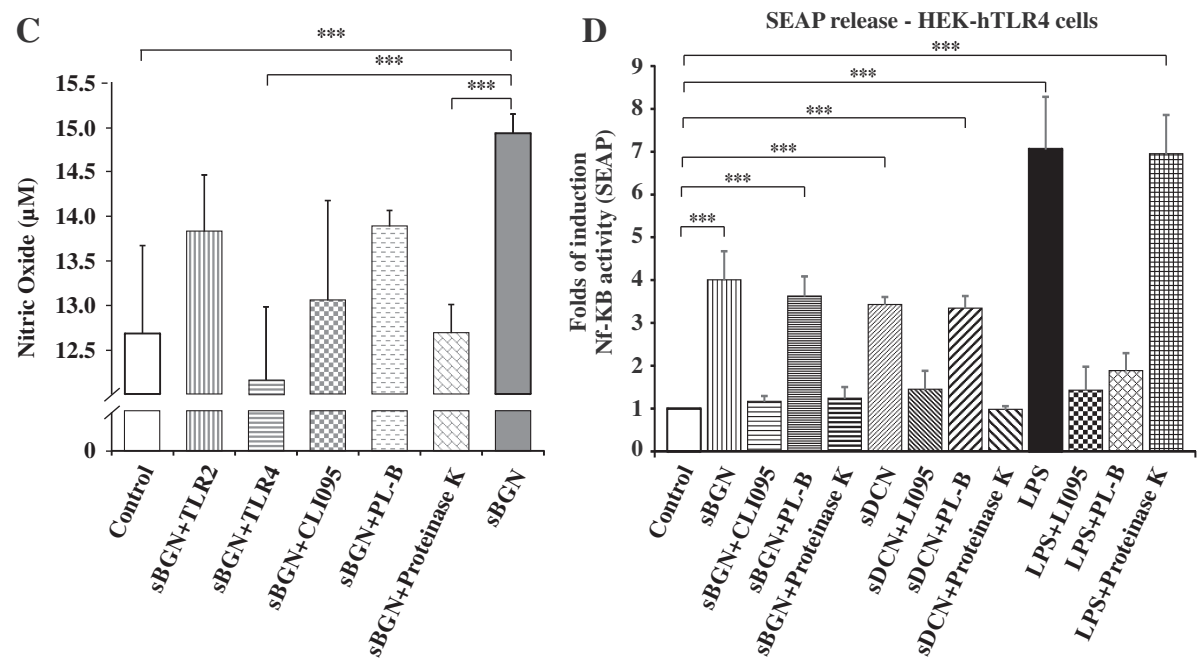

Fig. 8 Effects of soluble biglycan (sBGN) on HEK-hTLR4 secreted embryonic alkaline phosphatase (SEAP) production and chondrocyte production of nitric oxide (NO) compared with control groups. a In primary chondrocytes, sBGN increased NO. Lipopolysaccharide (LPS) was used as a positive control. $\mathbf{b}$ In cartilage explants, sBGN increased NO only in grade I osteoarthritis (OA) without a difference between mild and severe OA. c Addition of polymyxin B had no effect on the sBGN-induced NO production, while proteinase K restored NO production to levels seen in controls, indicating that there was no endotoxin contamination of sBGN. Addition of Toll-like receptor 2 (TLR2)-neutralising antibody caused no significant reduction of sBGN-mediated NO production, while TLR4 neutralisation impaired NO production. Samples were run as technical duplicates, and each experiment was done using samples from at least six different donors (biological replicates). $\mathbf{d}$ Effect of sBGN, soluble decorin (sDCN) and LPS on TLR4 activity. An SEAP assay was used to determine nuclear factor (NF)-KB activity following sBGN, sDCN or LPS stimulation of HEK-hTLR4. The results are from three independent experiments. All results are expressed as mean \pm SD. ${ }^{* *} p<0.01,{ }^{* * *} p<0.001$ vs. non-stimulated controls 
evidence. In HEK-hTLR4, which can be activated only through TLR4, sBGN and $\mathrm{SDCN}$ induced the secretion of AP, a reporter of NF-kB activation. This increase was significantly inhibited by CLI-095, an inhibitor of TLR4 signalling. Furthermore, in primary chondrocytes, sBGN induced production of $\mathrm{NO}$, which was almost completely impaired by CLI-095, an inhibitor of TLR4 signalling, and also by TLR4-neutralising antibody. In contrast, addition of TLR2 antibody had no significant effect on sBGN-induced NO production (Fig. 8). The signalling of TLR4 has been shown to occur via NF- $k B$, which in turn is the principal inducer of iNOS $[39,40]$. Furthermore, NO has been implicated as an important pro-inflammatory mediator of inflammation in OA [41, 42]. Thus, the sBGN induction of secreted AP in HEK-hTLR4 cells and the NO production in chondrocytes suggests that sBGN can induce a chondrocyte-mediated inflammatory response in cartilage.

sBGN has been shown in earlier in vitro experiments to exert dose-dependent effects at least up to $80 \mu \mathrm{g} / \mathrm{ml}$ concentration on peritoneal macrophages [43]. Although sBGN concentrations used in vitro are somewhat higher than those now measured in SF, they are probably pathophysiologically relevant. Given the fact that BGN is a pericellular matrix proteoglycan, local concentrations in the vicinity of chondrocytes can be expected to be higher than in SF [44]. Moreover, lack of biomechanically cyclic compression of the cartilage explants causes a drop in interstitial fluid pressure, and therefore higher doses of sBGN are needed to ensure outside-in access. Nevertheless, current in vitro techniques cannot mimic in vivo conditions, and therefore the next step would be to replicate the findings of the present study in an OA animal model.

Interaction of sBGN with TLR4 (and possibly to a lesser extent the other sBGN receptor, $\mathrm{P} 2 \mathrm{X}_{7 / 4}$ ) led to increased production of aggrecanases and collagenolytic enzymes at the mRNA and protein levels [45]. After cleavage across the collagen triple helix, fragments undergo spontaneous helix-to-coil transition to gelatin at body temperature. Thus, the sBGN-induced proteoglycanases (ADAMTS-4/5), collagenases (MMP-13, cat K) and gelatinases (MMP-9) are able to degrade all the major components of the hyaline articular cartilage. The observed elevated production of IL- 6 and IL-8 levels induced by $\mathrm{SBGN}$ in chondrocytes may also lead to increase recruitment and influx of inflammatory cells such as neutrophils and macrophages [46, 47]. Together the observed levels of MMPs and cytokines add further molecular stress to the OA joint and might create positive feedback loop of inflammation and cartilage degradation.

Soluble BGN induced expression of proteinases clearly more effectively in grade I than in grade IV OA. Grade IV OA represents an advanced stage of the disease and the low response of grade IV OA to sBGN could represent functio laesa (i.e., function disturbed as a result of inflammation and loss of cartilage). Interestingly $B G N$ mRNA is more upregulated in advanced OA compared with early stages suggesting in fact that desensitisation to BGN in grade IV OA explants may have occurred due to long term exposure at the pericellular lacunae or by an attempt of the cartilage to compensate the proteoglycan loss [22]. In addition, TLR4 upregulation by sBGN was substantially higher in grade I OA vs grade IV OA. Thus as expected TLR4-mediated NF- $\mathrm{KB}$ pathway products (i.e. proteinases and aggrecanases) were also proportionally upregulated. Likewise $\mathrm{NO}$, a product of iNOS which is regulated by NF-kB pathway, was also increased in grade I OA vs. grade IV OA.

In primary chondrocytes $\mathrm{sBGN}$ inhibited synthesis of the collagen (fibre network) and aggrecans (ground substance). In relatively well preserved grade I OA explants sBGN had a slight stimulatory effects on matrix synthesis, whereas in more advanced grade IV OA Col-II synthesis decreased significantly. Such an imbalance between degradation and synthesis results in a net loss of cartilage [48, 49].

Due to their potential destructive properties, proteolytic enzymes are tightly regulated. ADAMTS-4/ADAMTS-5 and MMPs are regulated on the transcriptional level (e.g., by sBGN), as shown in the present study. They are synthesised as latent pro-enzymes, which for activation require proteolytic removal of the activation (pro)peptide [50,51]. The MMPs are regulated by tissue inhibitors of metalloproteinases. Therefore, the most direct way to demonstrate sBGN-induced proteolysis of cartilage is to demonstrate a release of proteoglycan and collagen degradation products. This was done by using GAG and collagen-binding dyes and light absorption. Proteoglycan release was statistically significant only in grade I OA, as expected given the substantially higher catabolic response by grade I OA chondrocytes. In contrast, collagen release was significant only in type IV OA, perhaps because collagen becomes accessible for collagenolytic enzymes only after proteoglycan depletion [52]. Release of proteoglycans was confirmed by staining, which revealed their depletion from cartilage matrix in partly overlapping samples.

\section{Conclusions}

We demonstrate that intact $\mathrm{sBGN}$ is present in knee SF of patients with advanced knee OA or RA, whereas only low amounts of sDCN could be detected. sBGN upregulates TLR4 expression in chondrocytes, increases the expression and concentration levels of catabolic factors and decreases the expression of anabolic factors, resulting in net loss of cartilage. The mechanism of action of sBGN in chondrocytes is mediated mainly through activation of TLR4. These results support the importance TLR4 signalling in OA pathomechanisms, and thus TLR4 may be a potential 
therapeutic target in inflammation- and catabolismmediated cartilage degenerative disorders. Importantly, our findings strongly support the role of sBGN, a major ECM protein, as a potential biomarker, therapeutic target and contributor in the catabolic events that occur during progression of OA.

\begin{abstract}
Abbreviations
ACAN: aggrecan; ADAMTS: aggrecanase; AP: alkaline phosphatase; Col-II: collagen type II; CTSK: cathepsin K; DMEM: Dulbecco's modified Eagle's medium; ECM: extracellular matrix; ELISA: enzyme-linked immunosorbent assay; FCS: foetal calf serum; GAPDH: glyceraldehyde 3-phosphate dehydrogenase; IL: interleukin; iNOS: inducible nitric oxide synthase; LPS: lipopolysaccharide; MMP: matrix metalloproteinase; mRNA: messenger RNA; NF-KB: nuclear factor-kB; NO: nitric oxide; OA: osteoarthritis; PBS: phosphate-buffered saline; RA: rheumatoid arthritis; sBGN: soluble biglycan; sDCN: soluble decorin; SEAP: secreted embryonic alkaline phosphatase; SF: synovial fluid; SGAG: sulphated glycosaminoglycan; SLRP: small leucine-rich proteoglycan; TBP: TATA box-binding protein; TKA: total knee arthroplasty; TLR: Toll-like receptor.
\end{abstract}

\section{Competing interests}

The authors declare that they have no competing interests.

\section{Authors' contributions}

BG participated in the conception and design of the study, cell culture functional studies and data analysis, interpretation of data and manuscript writing. SA performed chondrocyte isolation and participated in SGAG and collagen biochemical assay methods and analysis as well as manuscript writing. YP acquired and interpreted data and participated in manuscript. SJ performed surgical removal of cartilage and collection of synovial fluid, data interpretation and manuscript writing. KY participated in the conception and design of the study, interpretation of data and manuscript writing. ND participated in the conception and design of the study, interpretation of data and manuscript writing. EK participated in the conception and design of the study, interpretation of data and manuscript writing. All authors read and approved the final manuscript.

\section{Acknowledgements}

We acknowledge JPV Sainio for his valuable contribution in helping with cell culture experiments and RT-PCR analysis. This work was supported by ORTON Orthopaedic Hospital of the Invalid Foundation, the Finnish Medical Society, Maire Lisko Foundation, the Finnish Society for Rheumatology, the Medcare Foundation and Helsinki University Central Hospital funds.

\section{Author details}

'Department of Internal Medicine and Rehabilitation, University of Helsinki and Helsinki University (Central) Hospital, Biomedicum 1, PO Box 63, FIN-00290 Helsinki, Finland. ${ }^{2}$ Department of Rheumatology, University of Helsinki and Helsinki University (Central) Hospital, Helsinki, Finland. ${ }^{3}$ ORTON Orthopaedic Hospital, Helsinki, Finland.

Received: 17 June 2015 Accepted: 14 December 2015

Published online: 24 December 2015

\section{References}

1. Woolf AD. The bone and joint decade: strategies to reduce the burden of disease: the Bone and Joint Monitor Project. J Rheumatol Suppl. 2003;67:6-9.

2. Felson DT. Osteoarthritis: priorities for osteoarthritis research: much to be done. Nat Rev Rheumatol. 2014;10:447-8.

3. Berenbaum F. Osteoarthritis as an inflammatory disease (osteoarthritis is not osteoarthrosis!). Osteoarthritis Cartilage. 2013;21:16-21.

4. Gao Y, Liu S, Huang J, Guo W, Chen J, Zhang L, et al. The ECM-cell interaction of cartilage extracellular matrix on chondrocytes. Biomed Res Int. 2014:2014:648459.

5. Troeberg L, Nagase H. Proteases involved in cartilage matrix degradation in osteoarthritis. Biochim Biophys Acta. 2012;1824:133-45.

6. Kim HA, Cho ML, Choi HY, Yoon CS, Jhun JY, Oh HJ, et al. The catabolic pathway mediated by Toll-like receptors in human osteoarthritic chondrocytes. Arthritis Rheum. 2006;54:2152-63.
7. Johnson GB, Brunn GJ, Kodaira Y, Platt JL. Receptor-mediated monitoring of tissue well-being via detection of soluble heparan sulfate by Toll-like receptor 4. J Immunol. 2002;168:5233-9.

8. Chang MY, Tanino Y, Vidova V, Kinsella MG, Chan CK, Johnson PY, et al. A rapid increase in macrophage-derived versican and hyaluronan in infectious lung disease. Matrix Biol. 2014;34:1-12.

9. Okamura Y, Watari M, Jerud ES, Young DW, Ishizaka ST, Rose J, et al. The extra domain A of fibronectin activates Toll-like receptor 4. J Biol Chem. 2001;276:10229-33.

10. Campo GM, Avenoso A, Campo S, D'Ascola A, Nastasi G, Calatroni A. Molecular size hyaluronan differently modulates Toll-like receptor-4 in LPSinduced inflammation in mouse chondrocytes. Biochimie. 2010;92:204-15.

11. Schelbergen RFP, Blom AB, van den Bosch MHJ, Slöetjes A, AbdollahiRoodsaz S, Schreurs BW, et al. Alarmins S100A8 and S100A9 elicit a catabolic effect in human osteoarthritic chondrocytes that is dependent on Toll-like receptor 4. Arthritis Rheum. 2012;64:1477-87.

12. Gay NJ, Gangloff M, Weber AN. Toll-like receptors as molecular switches. Nat Rev Immunol. 2006;6:693-8.

13. Roughley PJ. The structure and function of cartilage proteoglycans. Eur Cell Mater. 2006;12:92-101.

14. Anders HJ, Schaefer L. Beyond tissue injury—damage-associated molecular patterns, Toll-like receptors, and inflammasomes also drive regeneration and fibrosis. J Am Soc Nephrol. 2014;25:1387-400.

15. Song R, Zeng Q, Ao L, Yu JA, Cleveland JC, Zhao KS, et al. Biglycan induces the expression of osteogenic factors in human aortic valve interstitial cells via Toll-like receptor-2. Arterioscler Thromb Vasc Biol. 2012;32:2711-20.

16. Moreth K, Frey H, Hubo M, Zeng-Brouwers J, Nastase MV, Hsieh LT, et al. Biglycan-triggered TLR-2- and TLR-4-signaling exacerbates the pathophysiology of ischemic acute kidney injury. Matrix Biol. 2014;35:143-51.

17. Moreth K, Brodbeck R, Babelova A, Gretz N, Spieker T, Zeng-Brouwers J, et al. The proteoglycan biglycan regulates expression of the B cell chemoattractant CXCL13 and aggravates murine lupus nephritis. J Clin Invest. 2010:120;4251-72.

18. Merline R, Moreth K, Beckmann J, Nastase MV, Zeng-Brouwers J, Tralhão JG, et al. Signaling by the matrix proteoglycan decorin controls inflammation and cancer through PDCD4 and microRNA-21. Sci Signal. 2011;4:ra75.

19. Blom $A B$, van den Berg WB. The synovium and its role in osteoarthritis. In: Bronner F, Farach-Carson MC, editors. Bone and osteoarthritis. London: Springer; 2007. p. 65-79.

20. $\mathrm{Ni} \mathrm{GX,} \mathrm{Li} \mathrm{Z,} \mathrm{Zhou} \mathrm{YZ.} \mathrm{The} \mathrm{role} \mathrm{of} \mathrm{small} \mathrm{leucine-rich} \mathrm{proteoglycans} \mathrm{in}$ osteoarthritis pathogenesis. Osteoarthritis Cartilage. 2014;22:896-903.

21. Polgar A, Falus A, Koo E, Ujfalussy I, Sesztak M, Szuts I, et al. Elevated levels of synovial fluid antibodies reactive with the small proteoglycans biglycan and decorin in patients with rheumatoid arthritis or other joint diseases. Rheumatology (Oxford). 2003;42:522-7.

22. Bock HC, Michaeli P, Bode C, Schultz W, Kresse H, Herken R, et al. The small proteoglycans decorin and biglycan in human articular cartilage of late-stage osteoarthritis. Osteoarthritis Cartilage. 2001;9:654-63.

23. Monfort J, Tardif G, Reboul P, Mineau F, Roughley P, Pelletier JP, et al. Degradation of small leucine-rich repeat proteoglycans by matrix metalloprotease-13: identification of a new biglycan cleavage site. Arthritis Res Ther. 2006;8:R26.

24. Melching LI, Fisher WD, Lee ER, Mort JS, Roughley PJ. The cleavage of biglycan by aggrecanases. Osteoarthritis Cartilage. 2006;14:1147-54.

25. Melrose J, Fuller ES, Roughley PJ, Smith MM, Kerr B, Hughes CE, et al. Fragmentation of decorin, biglycan, lumican and keratocan is elevated in degenerate human meniscus, knee and hip articular cartilages compared with age-matched macroscopically normal and control tissues. Arthritis Res Ther. 2008;10:R79.

26. Dougados M, Ayral X, Listrat V, Gueguen A, Bahuaud J, Beaufils P, et al. The SFA system for assessing articular cartilage lesions at arthroscopy of the knee. Arthroscopy. 1994;10:69-77.

27. Heinola T, Kouri VP, Clarijs P, Ciferska H, Sukura A, Salo J, et al. High mobility group box-1 (HMGB-1) in osteoarthritic cartilage. Clin Exp Rheumatol. 2010;28:511-8.

28. Kay J, Upchurch KS. ACR/EULAR 2010 rheumatoid arthritis classification criteria. Rheumatology (Oxford). 2012;51 Suppl 6:vi5-9.

29. Vlachakis D, Tsaniras SC, Feidakis C, Kossida S. Molecular modelling study of the 3D structure of the biglycan core protein, using homology modelling techniques. J Mol Biochem. 2013;2:85-93.

30. Bobacz K, Sunk IG, Hofstaetter JG, Amoyo L, Toma CD, Akira S, et al. Toll-like receptors and chondrocytes: the lipopolysaccharide-induced decrease in 
cartilage matrix synthesis is dependent on the presence of Toll-like receptor 4 and antagonized by bone morphogenetic protein 7. Arthritis Rheum. 2007;56:1880-93

31. Nastase MV, lozzo RV, Schaefer L. Key roles for the small leucine-rich proteoglycans in renal and pulmonary pathophysiology. Biochim Biophys Acta. 2014;1840:2460-70.

32. Frey H, Schroeder N, Manon-Jensen T, lozzo RV, Schaefer L. Biological interplay between proteoglycans and their innate immune receptors in inflammation. FEBS J. 2013;280:2165-79.

33. Young AA, Smith MM, Smith SM, Cake MA, Ghosh P, Read RA, et al. Regional assessment of articular cartilage gene expression and small proteoglycan metabolism in an animal model of osteoarthritis. Arthritis Res Ther. 2005;7:R852-61.

34. Monach PA, Hueber W, Kessler B, Tomooka BH, BenBarak M, Simmons BP, et al. A broad screen for targets of immune complexes decorating arthritic joints highlights deposition of nucleosomes in rheumatoid arthritis. Proc Natl Acad Sci U S A. 2009;106:15867-72.

35. Zvaifler NJ. Rheumatoid synovitis: an extravascular immune complex disease. Arthritis Rheum. 1974;17:297-305.

36. Nandakumar KS, Holmdahl R. Antibody-induced arthritis: disease mechanisms and genes involved at the effector phase of arthritis. Arthritis Res Ther. 2006;8:223.

37. Wang Q, Rozelle AL, Lepus CM, Scanzello CR, Song JJ, Larsen DM, et al. Identification of a central role for complement in osteoarthritis. Nat Med. 2011;17:1674-9.

38. Barreto G, Sillat T, Soininen A, Ylinen P, Salem A, Konttinen YT, et al. Do changing Toll-like receptor profiles in different layers and grades of osteoarthritis cartilage reflect disease severity? J Rheumatol. 2013;40:695-702.

39. Akira S, Takeda K. Toll-like receptor signalling. Nat Rev Immunol. 2004;4:499-511.

40. Feng X, Guo Z, Nourbakhsh M, Hauser H, Ganster R, Shao L, et al. Identification of a negative response element in the human inducible nitric-oxide synthase (hiNOS) promoter: The role of NF-kB-repressing factor (NRF) in basal repression of the hiNOS gene. Proc Natl Acad Sci U S A. 2002;99:14212-7.

41. Boileau C, Martel-Pelletier J, Moldovan F, Jouzeau JY, Netter P, Manning PT, et al. The in situ up-regulation of chondrocyte interleukin-1-converting enzyme and interleukin-18 levels in experimental osteoarthritis is mediated by nitric oxide. Arthritis Rheum. 2002;46:2637-47.

42. Pelletier JP, Martel-Pelletier J, Abramson SB. Osteoarthritis, an inflammatory disease: potential implication for the selection of new therapeutic targets. Arthritis Rheum. 2001:44:1237-47.

43. Babelova A, Moreth K, Tsalastra-Greul W, Zeng-Brouwers J, Eickelberg O, Young MF, et al. Biglycan, a danger signal that activates the NLRP3 inflammasome via Toll-like and P2X receptors. J Biol Chem. 2009;284:24035-48.

44. Miosge N, Flachsbart K, Goetz W, Schultz W, Kresse H, Herken R. Light and electron microscopical immunohistochemical localization of the small proteoglycan core proteins decorin and biglycan in human knee joint cartilage. Histochem J. 1994;26:939-45.

45. Knight MM, McGlashan SR, Garcia M, Jensen CG, Poole CA. Articular chondrocytes express connexin 43 hemichannels and $\mathrm{P} 2$ receptors $-\mathrm{a}$ putative mechanoreceptor complex involving the primary cilium? J Anat. 2009:214:275-83

46. Blom AB, van Lent PL, Holthuysen AE, van der Kraan PM, Roth J, van Rooijen $\mathrm{N}$, et al. Synovial lining macrophages mediate osteophyte formation during experimental osteoarthritis. Osteoarthritis Cartilage. 2004;12:627-35.

47. Blom $A B$, van Lent PL, Libregts $S$, Holthuysen $A E$, van der Kraan PM, van Rooijen $\mathrm{N}$, et al. Crucial role of macrophages in matrix metalloproteinasemediated cartilage destruction during experimental osteoarthritis: involvement of matrix metalloproteinase 3. Arthritis Rheum. 2007;56:147-57.

48. Mueller MB, Tuan RS. Anabolic/catabolic balance in pathogenesis of osteoarthritis: identifying molecular targets. PM R. 2011;3(6 Suppl 1):S3-11.

49. Yang S, Kim J, Ryu JH, Oh H, Chun CH, Kim BJ, et al. Hypoxia-inducible factor-2a is a catabolic regulator of osteoarthritic cartilage destruction. Nat Med. 2010;16:687-93.

50. Malfait AM, Arner EC, Song RH, Alston JT, Markosyan S, Staten N, et al. Proprotein convertase activation of aggrecanases in cartilage in situ. Arch Biochem Biophys. 2008;478:43-51.
51. Springman EB, Angleton EL, Birkedal-Hansen H, Van Wart HE. Multiple modes of activation of latent human fibroblast collagenase: evidence for the role of a Cys 73 active-site zinc complex in latency and a "cysteine switch" mechanism for activation. Proc Natl Acad Sci U S A. 1990;87:364-8.

52. Pratta MA, Yao W, Decicco C, Tortorella MD, Liu RQ, Copeland RA, et al. Aggrecan protects cartilage collagen from proteolytic cleavage. J Biol Chem. 2003;278:45539-45.

\section{Submit your next manuscript to BioMed Central and we will help you at every step:}

- We accept pre-submission inquiries

- Our selector tool helps you to find the most relevant journal

- We provide round the clock customer support

- Convenient online submission

- Thorough peer review

- Inclusion in PubMed and all major indexing services

- Maximum visibility for your research

Submit your manuscript at www.biomedcentral.com/submit
Biomed Central 